\title{
Retinal ganglion cell loss in an ex vivo mouse model of optic nerve cut is prevented by curcumin treatment
}

\author{
Lucia Buccarello (iD ${ }^{1,2 \bowtie}$, Jessica Dragotto ${ }^{1}$, Kambiz Hassanzadeh ${ }^{1,3}$, Rita Maccarone ${ }^{3}$, Massimo Corbo ${ }^{4}$ and Marco Feligioni (iD ${ }^{1,4}{ }^{凶}$
}

(c) The Author(s) 2021

Retinal ganglion cell (RGC) loss is a pathologic feature common to several retinopathies associated to optic nerve damage, leading to visual loss and blindness. Although several scientific efforts have been spent to understand the molecular and cellular changes occurring in retinal degeneration, an effective therapy to counteract the retinal damage is still not available. Here we show that eyeballs, enucleated with the concomitant optic nerve cut (ONC), when kept in PBS for $24 \mathrm{~h}$ showed retinal and optic nerve degeneration. Examining retinas and optic nerves at different time points in a temporal window of $24 \mathrm{~h}$, we found a thinning of some retinal layers especially RGC's layer, observing a powerful RGC loss after $24 \mathrm{~h}$ correlated with an apoptotic, MAPKs and degradative pathways dysfunctions. Specifically, we detected a time-dependent increase of Caspase- $3,-9$ and pro-apoptotic marker levels, associated with a strong reduction of BRN3A and NeuN levels. Importantly, a powerful activation of JNK, c-Jun, and ERK signaling (MAPKs) were observed, correlated with a significant augmented SUMO-1 and UBC9 protein levels. The degradation signaling pathways was also altered, causing a significant decrease of ubiquitination level and an increased LC3B activation. Notably, it was also detected an augmented Tau protein level. Curcumin, a powerful antioxidant natural compound, prevented the alterations of apoptotic cascade, MAPKs, and SUMO-1 pathways and the degradation system, preserving the RGC survival and the retinal layer thickness. This ex vivo retinal degeneration model could be a useful method to study, in a short time window, the effect of neuroprotective tools like curcumin that could represent a potential treatment to contrast retinal cell death.

Cell Death Discovery (2021)7:394; https://doi.org/10.1038/s41420-021-00760-1

\section{INTRODUCTION}

Retinal ganglion cell (RGC) loss [1] causes visual loss and blindness in several ocular pathologies. Retina is a neuronal tissue in which RGCs transfer the optical signals to the visual cortex in the brain which composes the images [2,3]. An optic nerve damage induces progressive RGC apoptosis leading to blindness [4]. Pre-clinical studies in the retinal degeneration area are mostly pursued in using animal models, like glaucoma [5-7], in which RGC death is reproduced by several approaches, including the transient increase of intraocular pressure (IOP), optic nerve cut (ONC), or genetic manipulation [8-10]. The animal models are useful tools to investigate neuroprotective pharmacological treatments for visual system preservation [11] as well as in in vitro experiments, mostly based on RGC primary cultures, to test potential therapeutic compounds [12-14]. However, both in vivo and in vitro models do not fully recapitulate the human disease and showing invasiveness and reproducibility [15].

In this article, we performed an ex vivo model of ONC to test the neuroprotective effect of curcumin. ONC is obtained by enucleating the eyeballs and keeping the eyes in phosphate-buffered saline (PBS) for several hours at $4{ }^{\circ} \mathrm{C}$. This causes a fast ( $24 \mathrm{~h}$ ) RGC layer degeneration and other ganglion cell layer $(\mathrm{GCL})$, inner plexiform layer (IPL), and inner nuclear layer (INL) thinning. So, this model might be useful for a preliminary screening of neuroprotective compounds with the advantage to reduce live animal pain and to be more physiological than in vitro approaches. Several molecular markers have been examined to study the degenerative processes such as the apoptotic pathway, already recognized as retinal degeneration marker [16-19], although the involvement of kinases and caspases has been quite controversial [20], and the pro/anti-apoptotic markers including caspases and BCL2 family, MAP-kinase pathways like JNK and ERK.

Recently, we reported that SUMO-1 plays an important role in the hyperactivation of JNK and Tau in a cellular model of oxidative stress and that curcumin, a strong antioxidant and antiinflammatory natural molecule, was able to re-equilibrate the JNK-SUMO-1-Tau axis [21]. Since the involvement of SUMO in retinal function has been already shown [22], SUMO-1ylation levels in our ex vivo model has been investigated. In addition, photoreceptors and RGCs are known to be highly vulnerable to oxidative stress as that reactive oxidative species (ROS) imbalance is involved in many retinal diseases [23, 24]. Interestingly, curcumin ameliorates the retinal progressive damage by regulating the antioxidant system [25] and could represent an alternative treatment in retinal diseases [26]. Thus, the protective effect of curcumin has been tested in our ex vivo model.

We found that after $24 \mathrm{~h}$ from ONC and eyes enucleation induces a strong RGC death, optic nerve damage, and decrease of retina layers thickness and that curcumin pre-treatment is able to counteract the occurring retinal degeneration.

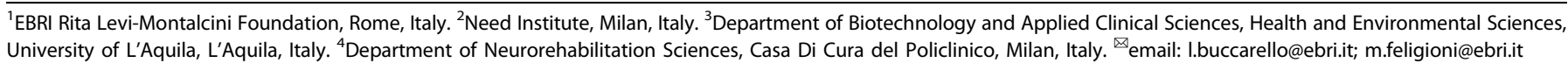




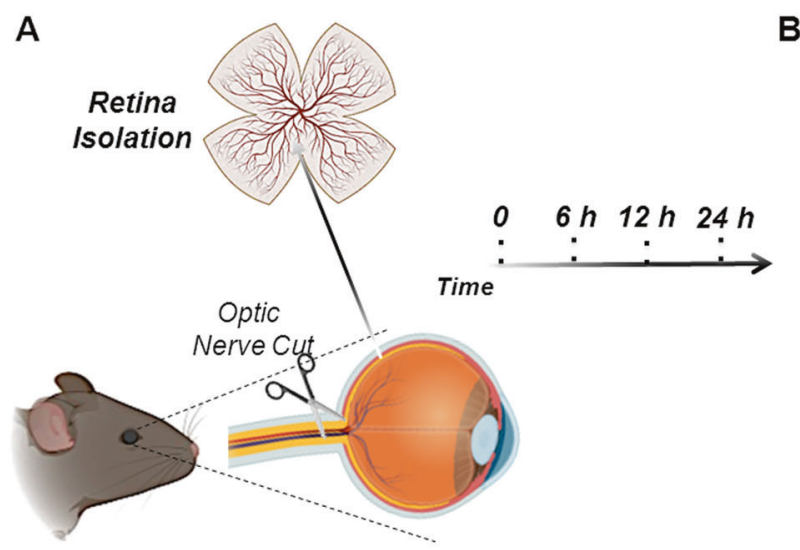

B

C
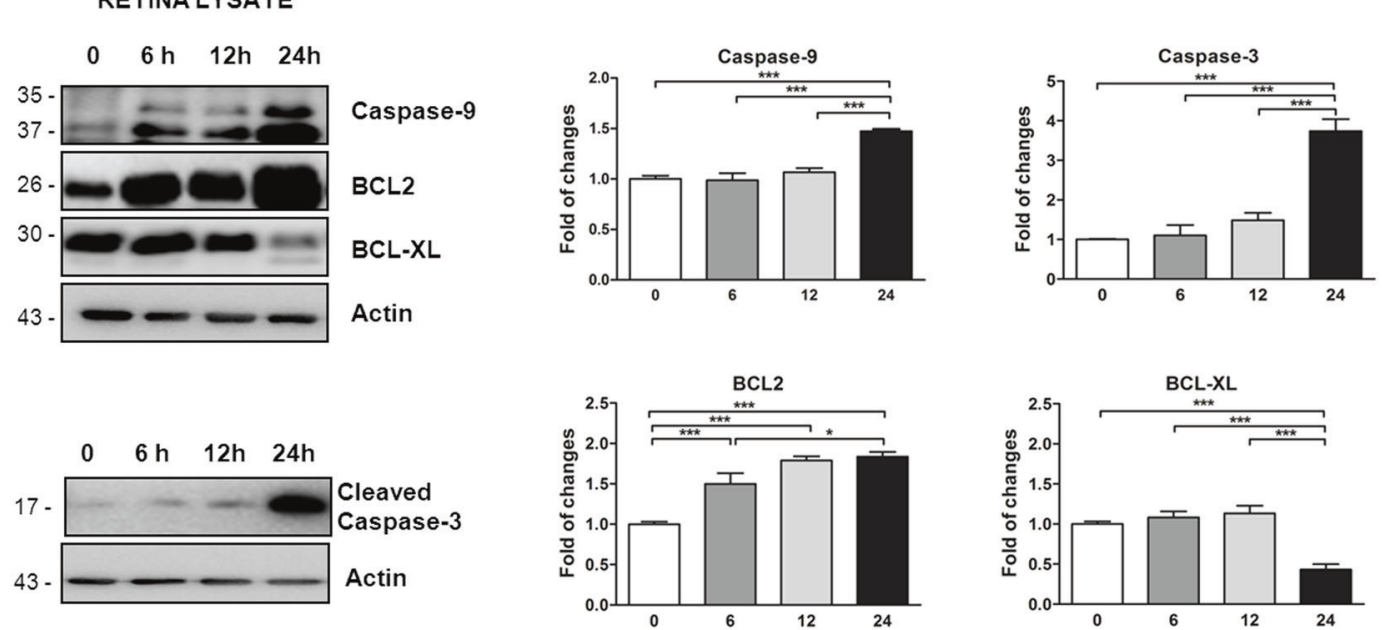

Fig. 1 Activation of apoptotic pathway in the optic nerve and retina of C57BL/6J mice after $24 \mathrm{~h}$ from eye removal. A Graphical representation of experimental scheme performed to study the time-dependent retina neurodegeneration. After the eyeballs enucleation, optic nerves and retinal dissections were collected immediately after the sacrifice (time 0), after 6 (time 6), 12 (time 12), and 24 (time 24) hours from the sacrifice. B Representative western blots and relative quantifications of optic nerve lysates collected immediately after eye removal (time 0), after $6 \mathrm{~h}$ (time 6), $12 \mathrm{~h}$ (time 12), and $24 \mathrm{~h}$ (time 24). Graphs showed the significant time-dependent increase of cleaved-Caspase-3 level and decreased NeuN level in optic nerve lysates collected at time 24 in comparison to time 0 . C Representative western blot and relative quantification of retinas lysates showed a significant augmented activation of Caspase-9, cleaved-Caspase-3, and BCL2 level at time 24 in comparison to time 0 . The BCL-XL level was decreased in retinas collected at time 24 in comparison to time 0 . Data were expressed as mean \pm SEM. One-way ANOVA, Tukey's post hoc test. ${ }^{*} P<0.05$, ${ }^{* *} P<0.01$, and ${ }^{* *} P<0.001[n=5]$.

\section{RESULTS}

The apoptotic pathway is activated in optic nerve and retina of enucleated mice eyeballs

Enucleated mouse eyeballs, including ONC, kept in PBS for $24 \mathrm{~h}$ develops a progressive retinal degeneration resulting from the parameters analyzed in the retinas collected at different time points (time $0: t 0 ; 6 \mathrm{~h}: t 6 ; 12 \mathrm{~h}: t 12$, and $24 \mathrm{~h}: t 24 ;$ Fig. $1 \mathrm{~A}$ ). Retinas and optic nerves lysates were examined by studying the apoptotic proteins, caspases, which are involved in RGC loss [27]. The cleaved-Caspase-3 level, active caspase effectors, was found increased twofolds, after $24 \mathrm{~h}$ from eye removal, in the optic nerve lysate $(1.91 \pm 0.06, P<0.001$ vs $t 0$; Fig. $1 \mathrm{~B})$ and a lower but significant increase at time $6(1.26 \pm 0.06, P<0.01$ vs t0; Fig. 1B) and $12(1.28 \pm 0.06, P<0.01$ vs t0; Fig. 1B). Coherently, the expression level of a neuronal marker, NeuN, was strongly decreased at $24 \mathrm{~h}$ only $(0.65 \pm 0.07, P<0.01$ vs t0; Fig. 1B). In parallel, in the retina lysates Caspase- 9 level, caspases initiator, was significantly increased in the retina lysates after $24 \mathrm{~h}(1.47 \pm$ $0.05, P<0.001$ vs t0; Fig. 1C). In line, a significant increase of cleaved-Caspase-3 level was observed at time $24(3.74 \pm 0.23, P<$ 0.001 vs t0; Fig. 1C).
Other two major anti-apoptotic proteins of the BCL2 family, $\mathrm{BCL} 2$ and $\mathrm{BCL}-\mathrm{XL}$, were also measured. They play important roles in inhibiting mitochondria-dependent extrinsic and intrinsic cell death pathways [28]. The BCL2 level was significantly augmented during the $24 \mathrm{~h}$ starting after time $6(1.50 \pm 0.09, P<0.001$ vs $t 0$; Fig. $1 C)$ and $12(1.79 \pm 0.09, P<0.001$ vs t0; Fig. $1 C)$ and reaching the maximal effect at time $24(1.84 \pm 0.08, P<0.001$ vs t0; Fig. 1C). In contrast, the BCL-XL level was decreased at time $24(0.43 \pm 0.08$, $P<0.001$ vs t0; Fig. $1 C)$, underlying an important alteration of the anti-apoptotic pathway in retinas extracted after $24 \mathrm{~h}$ after eye removal.

\section{Retinal cells survival reduction after $\mathbf{2 4} \mathrm{h}$ from mouse eyes removal}

Retinal cells vitality within 24 after ONC was evaluated by the expression level of the neuronal marker NeuN that was significantly reduced ( $t 24: 0.72 \pm 0.07, P<0.01$ vs $t 0$; Fig. $2 A$ ), while it was slightly increased at time 6 but not significant.

Moreover, the expression level of BRN3A, a specific marker for RGC nuclei, decreased in the retinas ( $t 6: 0.67 \pm 0.06, P<0.001$ vs $t 0$; $t 12: 0.67 \pm 0.06, P<0.001$ vs $t 0 ; t 24: 0.62 \pm 0.05, P<0.001$ vs $t 0$; 
A
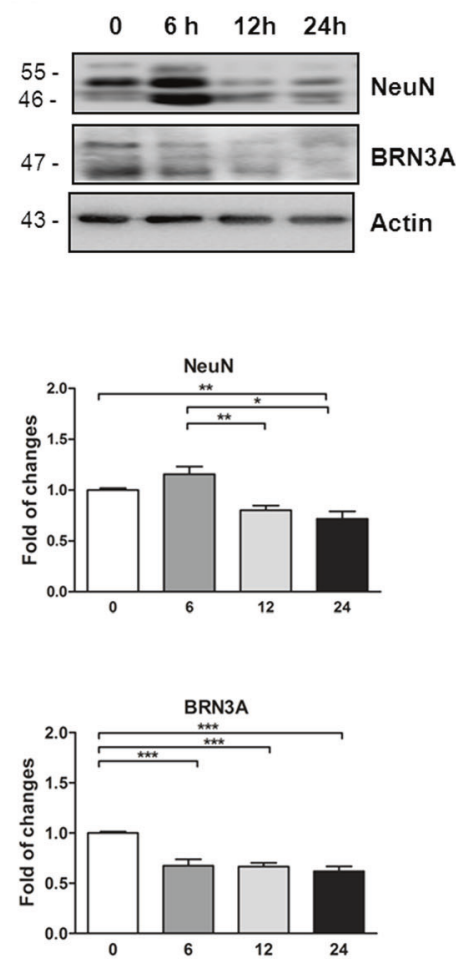

B

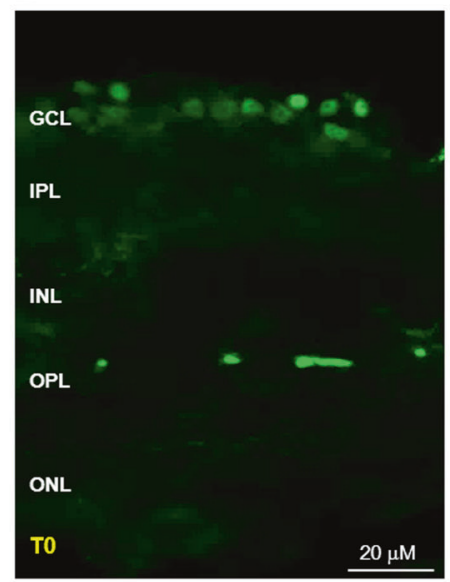

E

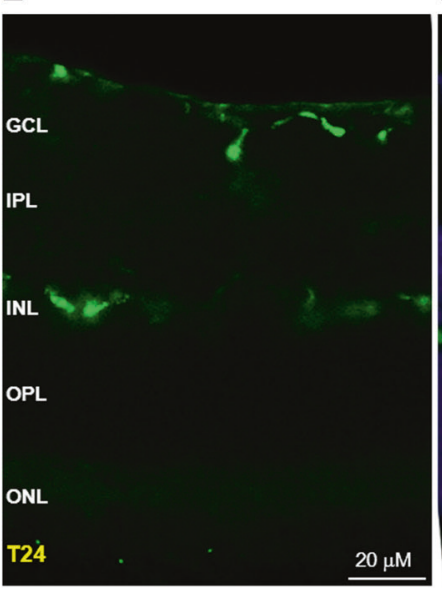

C

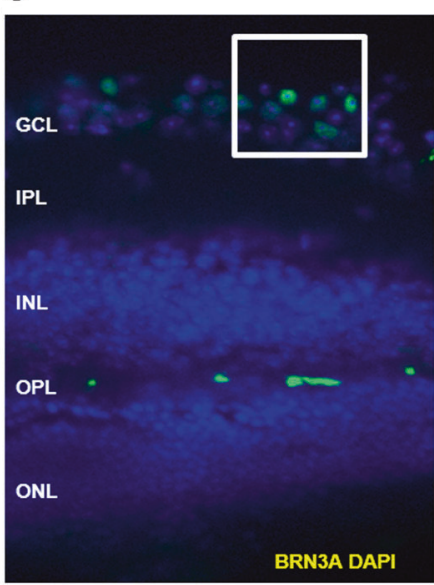

F

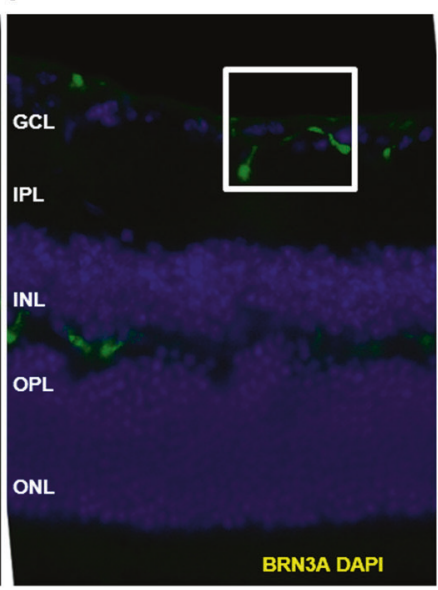

D

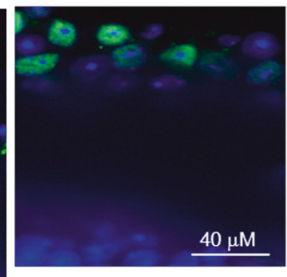

G

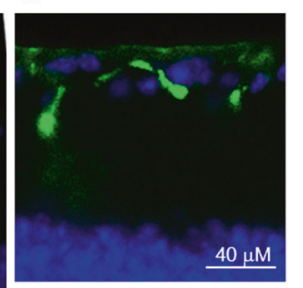

Fig. 2 Reduction of retinal ganglion cells and retinal cells vitality after $24 \mathrm{~h}$ from C57BL/6J mice eye removal. A Representative western blot and relative quantification of retinas lysates showed a significant reduction of BRN3A [a specific marker for RGC nuclei] and NeuN level in retina lysates collected at time 24 in comparison to time 0 . Data were expressed as mean \pm SEM. One-way ANOVA, Tukey's post hoc test. ${ }^{*} P<$ $0.05,{ }^{*} P<0.01$, and ${ }^{* *} P<0.001[n=5]$. B-G Immunofluorescence of retinal sections performed with BRN3A showed a reduction of BRN3Apositive cells (labeled in green) in retinas collected at time 24 (E-G) vs time 0 (B-D). Nuclei were stained with the nuclear marker DAPI (blue). GCL ganglion cell layer, IPL inner plexiform layer, INL inner nuclear layer, OPL outer plexiform layer, ONL outer nuclear layer. Scale bar: $20 \mu \mathrm{m}$. D, G Merged images at time 0 (D) and time 24 (G). D, G High magnification of merged images. Scale bar: $40 \mu \mathrm{m}$.

Fig. 2A). In parallel, the qualitative immunofluorescence images of retinal slices showed a reduction of BRN3a positive after $24 \mathrm{~h}$ (in green, Fig. 2E-G) (Fig. 2 B-D).

\section{MAPK pathways time-dependent changes and Tau expression \\ level}

The MAPK (mitogen-activated protein kinase) signaling pathways, c-Jun, N-terminal kinases (JNKs), and extracellular signal-regulated kinases (ERKs), play a critical role in the control of autophagy and apoptosis responses [29]. In line with data reported in literature [30-32], our results showed a clear activation of the apoptotic pathway in the retina.

JNK was strongly activated (as p-JNK/JNK) at time $24(1.46 \pm$ $0.08, P<0.001$ vs t0; Fig. $3 \mathrm{~A}$ ) but significantly reduced at time 6 $(0.24 \pm 0.09, P<0.001$ vs t0; Fig. $3 \mathrm{~A})$ and $12(0.33 \pm 0.09, P<0.001$ vs t0; Fig. $3 \mathrm{~A}$ ). Consequently, the phosphorylation of c-Jun, a transcription factor activated downstream by JNK, increased at time $24(2.11 \pm 0.12 ; P<0.001$ vs t0; Fig. 3B). Instead, ERK (as $p$ ERK/ERK) was slightly reduced at time $24(0.71 \pm 0.09, P<0.05$ vs t0; Fig. 3C). Tau accumulation in the visual system is an early phenomena of the retinal neuronal damage [18, 33] and interestingly it has a strong crosstalk with JNK in both physiological and pathological conditions [21]. Thus, Tau phosphorylation (as p-Tau/Tau) was analyzed and significantly augmented in retinas extracted at time $24(1.42 \pm 0.07, P<0.001$ vs $t 0$; Fig. 3D).

\section{SUMO-1ylation and degradation signaling pathways are altered in the ex vivo model}

SUMOylation is known to play an important role in retinal function $[22,34]$ and in oxidative stress [21]. Interestingly, SUMO-1ylation was highly increased in retinas at time $24(2.25 \pm 0.16, P<0.0001$ vs $t 0$; Fig. $3 E$ ) and the expression level of UBC9, the fundamental SUMOylation enzyme [35], was augmented in a time-dependent manner. Specifically, UBC9 level was increased at time point 12 and 24 ( $t 12: 2.37 \pm 0.22$ and $t 24: 2.77 \pm 0.19, P<0.0001$ vs $t 0$; Fig. 3E).

The failure of the degradation pathway is reported to be involved in several degenerative processes leading to retinal cell dysfunction in eye's pathologies [36]. Therefore, the ubiquitin and LC3B conversion level was measured in retina homogenates and ubiquitin level was reduced at time $6(0.61 \pm 0.11, P<0.05$ vs t0; Fig. 3F), at time $12(0.49 \pm 0.11, P<0.01$ vs t0; Fig. 3F), and time 24 $(0.59 \pm 0.09, P<0.01$ vs t0; Fig. $3 \mathrm{~F})$, while LC3B conversion (LC3BII/ 
A

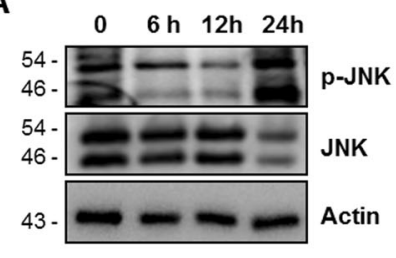

C

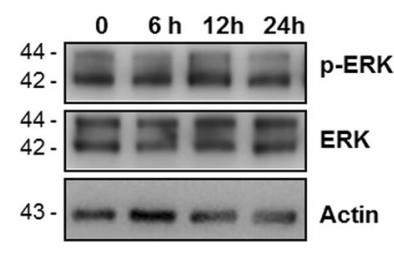

E

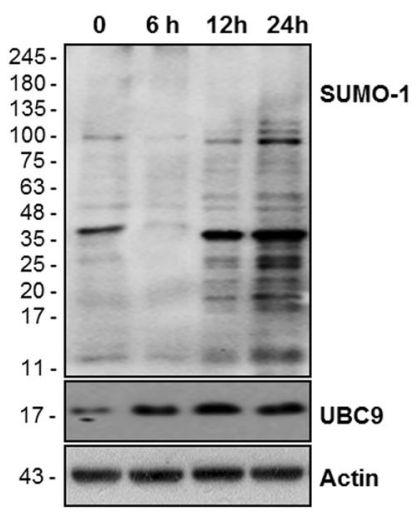

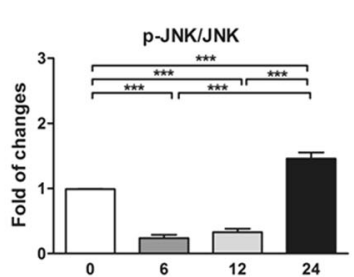
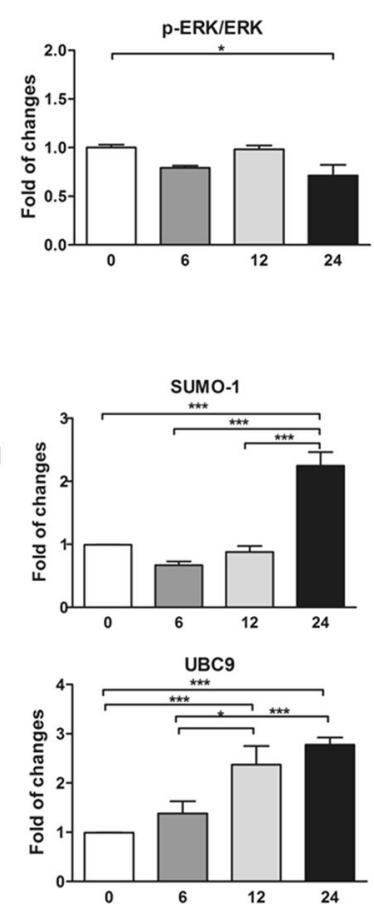

B
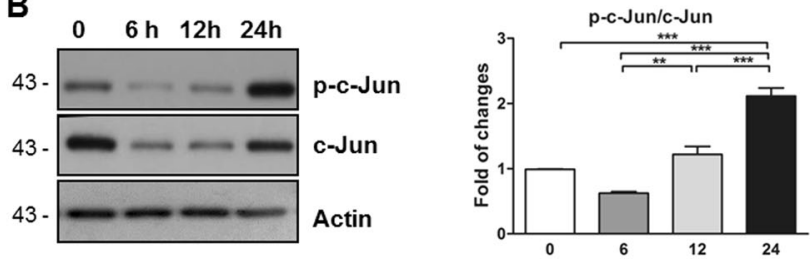

D
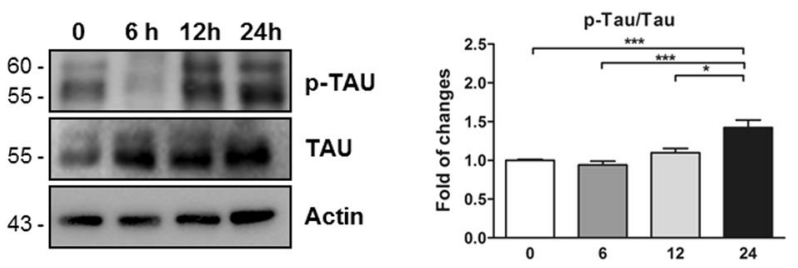

$\mathbf{F}$
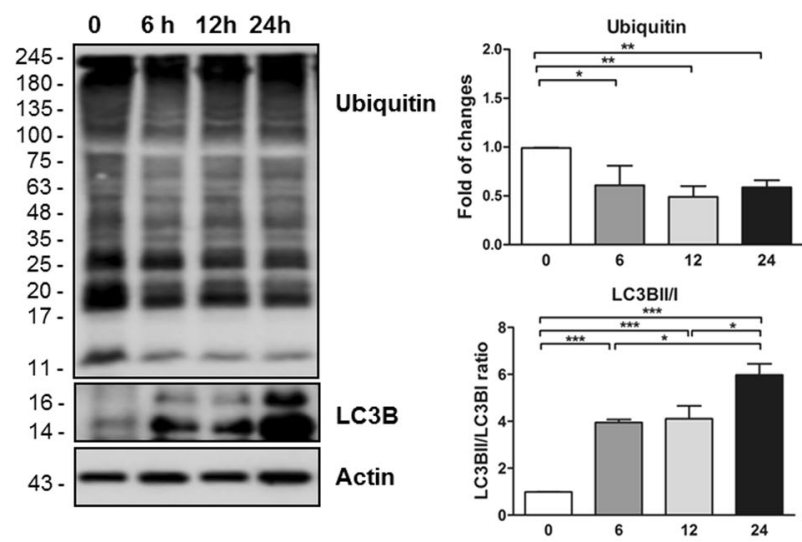

Fig. 3 MAPK pathways activation in the retina after $24 \mathrm{~h}$ from C57BL/6J mice eye removal. A-D Representative western blots and relative quantifications showed the significant higher p-JNK/JNK (A), p-c-Jun/c-Jun (B), and p-Tau/Tau (D) ratios in retina homogenates collected at time 24 in comparison to time 0, while the p-ERK/ERK (C) ratio was lower. E Representative western blots and relative quantifications showed the significant higher SUMO-1 and UBC9 level in retina homogenates collected at time 24 in comparison to time 0 . F Representative western blots and relative quantifications showed the significant time-dependent decrease of the ubiquitin level and increase of the LC3B level expressed as the LC3BII/I ratio in retina homogenates collected at time 24 in comparison to time $0 .{ }^{*} P<0.05,{ }^{* *} P<0.01,{ }^{* * *} P<0.001$, and ${ }^{* * * *} P$ $<0.0001[n=5]$. Data were expressed as mean \pm SEM. One-way ANOVA, Tukey's post hoc test.

LC3BI ratio) was augmented in each time points: time $6(3.95 \pm$ $0.44, P<0.0001$ vs $t 0$; Fig. 3 F), time $12(4.11 \pm 0.44, P<0.0001$ vs $t 0$; Fig. 3F), and time 24 (5.98 $\pm 0.44, P<0.0001$ vs t0, Fig. 3F).

\section{Curcumin treatment prevented RGC loss in our ex vivo model} Curcumin, a natural antioxidant compound largely used to contrast degenerative processes [21,37], was tested at 5 and $10 \mu \mathrm{M}$ to counteract the cell death of retinal layers in our ex vivo model at $24 \mathrm{~h}$. Firstly, we verified if curcumin vehicle (DMSO) had toxic effects and likely the levels of cleaved-Caspase-3, BAX, BCL2, and $B C L-X L$ were not altered at time 24 (see Suppl. Fig. 1A).

The immunofluorescence images showed a reduction of NeuN staining at time 24 (labeled in green, Fig. 4A, B) in which the number of NeuN-positive RGCs (Fig. $4 A^{\prime}-B^{\prime}$ ) were decreased $(25.4 \pm 1.5$ vs $11.80 \pm 1.5, P<0.001$; Fig. $4 \mathrm{E})$.

The pre-treatment with curcumin reduced RGC loss at $5 \mu \mathrm{M}$ $(19.2 \pm 1.3, P<0.001$ vs $t 24$; Fig. $4 \mathrm{E})$ and $10 \mu \mathrm{M}(20.4 \pm 1.3, P<$ 0.0001 ; Fig. 4E), shown also in the immunofluorescence images (labeled in green, Fig. $\left.4 C-C^{\prime}, D-D^{\prime}\right)$. Importantly, the NeuN expression level was reduced after $24 \mathrm{~h}(0.72 \pm 0.04, P<0.01$ vs t0; Fig. 4F) while curcumin pre-treatment prevented neuronal loss at both 5 and $10 \mu \mathrm{M}(5 \mu \mathrm{M}: 2.23 \pm 0.18 ; 10 \mu \mathrm{M}: 1.72 \pm 0.18, P<$ 0.0001 vs t24; Fig. 4F).
Curcumin protected the thickness of some retinal cells layer in our ex vivo model

The neuroprotective curcumin effect was analyzed by measuring the thickness of the retinas layers (Fig. 5A-E). Interestingly, the reduction of GCL thickness confirmed RGC loss at time 24 (18.62 \pm 4.70; $P<0.05$ vs t0; Fig. 5C, F). The curcumin $5 \mu \mathrm{M}(27.13 \pm 5.02$, Fig. 5D, F) and $10 \mu \mathrm{M}(26.73 \pm 5.02$, Fig. 5E, F) pre-treatment induced a slight, but not significant, recovery of RGC loss within the $24 \mathrm{~h}$. The IPL thickness was reduced at $24 \mathrm{~h}(78.10 \pm 7.92 ; P<$ 0.05 vs $t 0$; Fig. $5 \mathrm{C}, \mathrm{G}$ ) and the IPL thinning was not prevented by $5 \mu \mathrm{M}$ curcumin treatment $(77.35 \pm 8.40, P<0.05$ vs $t 0$; Fig. $5 \mathrm{D}, \mathrm{G})$, while $10 \mu \mathrm{M}$ induced a slight protection $(99.25 \pm 8.40$, Fig. $5 \mathrm{E}, \mathrm{G})$. The INL and outer plexiform (OPL) layer thicknesses were not affected within the $24 \mathrm{~h}$. Interestingly, the outer nuclear layer $(\mathrm{ONL})$ thickness was slightly reduced in $24 \mathrm{~h}(114.9 \pm 6.50, P<0.01$ vs t0; Fig. $5 \mathrm{C}, \mathrm{J})$, prevented with $10 \mu \mathrm{M}$ curcuma treatment $(149.6 \pm 6.50, P<0.01$ vs $t 24$; Fig. $5 \mathrm{E}, \mathrm{J})$.

\section{Curcumin prevented apoptotic pathways and MAPKs activation in the ex vivo model}

Interestingly, curcumin pre-treatment prevented the activation of the apoptotic pathway in retinal lysates, specifically the expression level of Caspase- $9(0.73 \pm 0.15, P<0.001$ vs $t 24$; Fig. $6 \mathrm{~A})$ and 
A

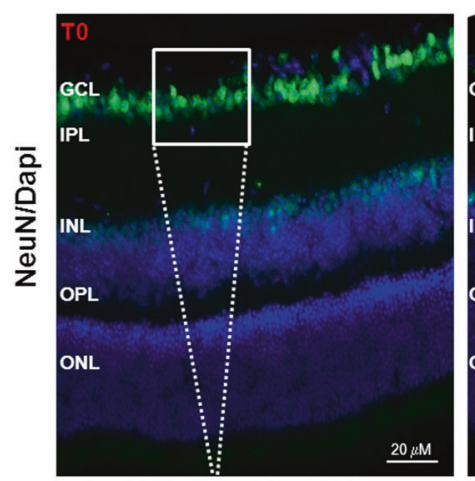

$A^{\prime}$

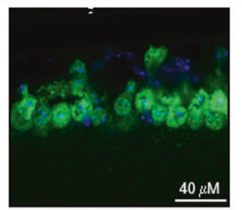

B

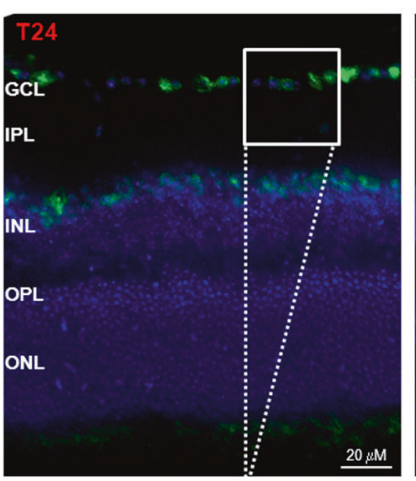

$B^{\prime}$

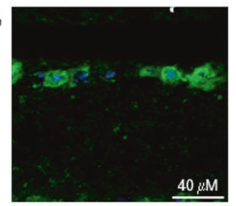

C

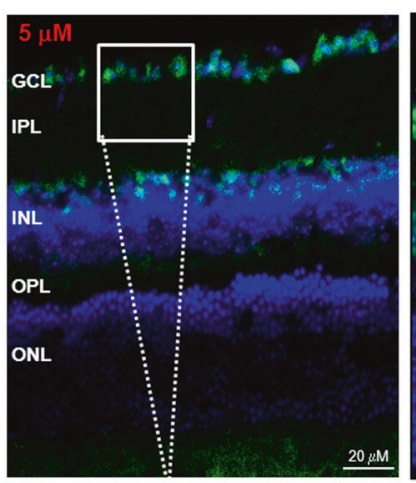

$c^{\prime}$

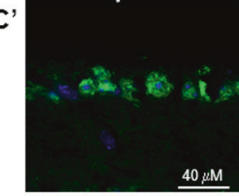

D

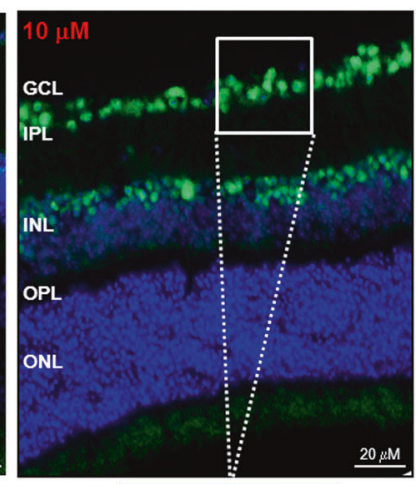

D'

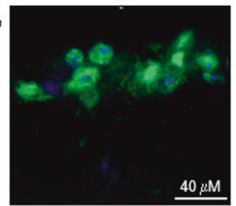

E

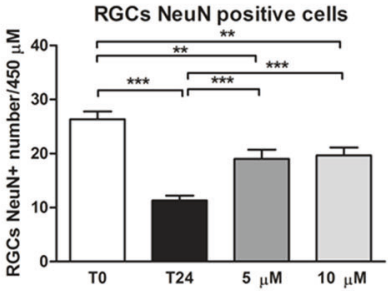

F

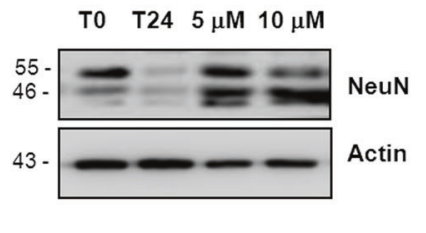

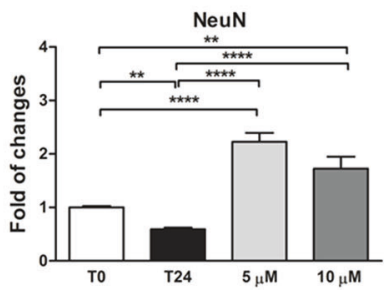

Fig. 4 Curcumin treatment reduces the retinal degeneration preventing the RGC loss in our ex vivo model of retinal degeneration. A-D Immunofluorescence analysis of retinal sections collected immediately after the sacrifice (T0), after $24 \mathrm{~h}$ after the sacrifice (T24), $24 \mathrm{~h}$ pretreated with $5 \mu \mathrm{M}(5 \mu \mathrm{M})$ and $10 \mu \mathrm{M}(10 \mu \mathrm{M})$ of curcumin. IF images indicated a decrease of RGC NeuN-positive cells (green dots) in samples collected after $24 \mathrm{~h}$ from eye removal (B-B') compared to time $0\left(\mathbf{A}-\mathbf{A}^{\prime}\right)$. The $5 \mu \mathrm{M}\left(\mathbf{C}-\mathbf{C}^{\prime}\right)$ as well as $10 \mu \mathrm{M}\left(\mathbf{D}-\mathbf{D}^{\prime}\right)$ of curcumin treatment was able to prevent the RGC loss detected at time 24. Nuclei were stained with the nuclear marker DAPI (blue). Scale Bar: $20 \mu \mathrm{m} . \mathbf{A}^{\prime}, \mathbf{B}^{\prime}, \mathbf{C}^{\prime}, \mathbf{D}^{\prime}$ High magnification of merged images. Scale bar: $40 \mu \mathrm{m}$. GCL ganglion cell layer, IPL inner plexiform layer, INL inner nuclear layer, OPL outer plexiform layer, ONL outer nuclear layer. E Quantification of the RGCs NeuN-positive (NeuN+) number showed a reduction of RGC NeuN+ in retinas collected at time 24 compared to time 0 . Both 5 and $10 \mu \mathrm{M}$ curcumin administration was able to reduce RGC loss, increasing significantly RGC NeuN + cells number. $\mathbf{F}$ Representative western blot and relative densitometric quantification showed a reduction of NeuN level in retina homogenates collected at time 24 and a significant increased NeuN level induced by the 5 and $10 \mu \mathrm{M}$ curcumin administrations. ${ }^{* *} P<0.01$ and ${ }^{* * * *} P<0.0001[n=5]$. Data were expressed as mean \pm SEM. One-way ANOVA, Tukey's post hoc test.

Caspase-3 $(0.165 \pm 0.45 ; P<0.0001$ vs $t 24$, Fig. $6 \mathrm{~A})$ were both reduced at $5 \mu \mathrm{M}$ of curcumin, while at $10 \mu \mathrm{M}$ the Caspase- 9 level was decreased $(0.49 \pm 0.15, P<0.0001$ vs $t 24$; Fig. $6 \mathrm{~A})$ but not the level of cleaved-Caspase-3. This effect is in line with previous studies indicating that $5 \mu \mathrm{M}$ of curcumin was the most effective dose against degenerative processes $[21,37,38]$. In fact, $10 \mu \mathrm{M}$ of curcumin was unable to contrast the BCL2 and BAX levels augmentation at time $24(\mathrm{BCL} 2: 1.77 \pm 0.12 ; P<0.001$ vs t0 and BAX: $2.60 \pm 0.18, P<0.0001$ vs t0; Fig. $6 \mathrm{~A})$. However, $5 \mu \mathrm{M}$ of curcumin reduced $B C L 2$ and $B A X$ levels $(B C L 2: 1.36 \pm 0.12, P<0.05$ vs $t 24 ; B A X: 1.83 \pm 0.18, P<0.01$ vs $t 24 ;$ Fig. $6 A)$. In line, the $B C L-X L$ level was decreased at time $24(0.44 \pm 0.09, P<0.0001$ vs $t 0$; Fig. $6 \mathrm{~A})$ and the pre-treatment with $5 \mu \mathrm{M}$ of curcumin contrasted this effect $(1.37 \pm 0.09, P<0.001$ vs $t 24$; Fig. $6 \mathrm{~A})$, while $10 \mu \mathrm{M}$ of curcumin was again ineffective.

Then, JNK phosphorylation was augmented at time $24(1.54 \pm$ $0.17, P<0.05$; Fig. $6 \mathrm{~B})$, but surprisingly the pre-treatment with $5 \mu \mathrm{M}$ of curcumin caused an even more significant increase of $\mathrm{p}$ JNK/JNK ratio $(2.36 \pm 0.17, P<0.01$ vs $t 24$; Fig. $6 \mathrm{~B})$, while $10 \mu \mathrm{M}$ of curcumin showed a slight, but not significant, reduction of JNK activation (1.22 \pm 0.17 , Fig. $6 \mathrm{~B})$.

C-Jun activity was strongly increased at time $24(2.11 \pm 0.12, P<$ 0.001 vs t0; Fig. 6B) and it was reduced by both doses of curcumin (5 $\mu \mathrm{M}: 1.24 \pm 0.11, P<0.001 ; 10 \mu \mathrm{M}: 0.68 \pm 0.11, P<0.0001$ vs $t 24$;
Fig. 6B). As expected, ERK activation had an opposite trend respect to JNK and c-Jun showing a decrement of $p$-ERK/ERK ratio at time 24 that was reverted by both $5 \mu \mathrm{M}(2.03 \pm 0.14, P<0.0001$ vs $t 24$; Fig. $6 \mathrm{~B})$ and $10 \mu \mathrm{M}(2.10 \pm 0.15, P<0.0001$ vs $t 24$; Fig. $6 \mathrm{~B})$ of curcumin.

Finally, we found that the augmented $\mathrm{p}$-Tau/Tau ratio level caused by the $24 \mathrm{~h}$ after eyeballs enucleation $(1.94 \pm 0.16, P<0.01$ vs $t 0$; Fig. $6 \mathrm{~B}$ ) was significantly prevented by the pre-treatment of curcumin with by $10 \mu \mathrm{M}$ of curcumin $(0.83 \pm 0.16, P<0.0001$ vs t24; Fig. 6B).

\section{Curcumin influenced SUMO-1 activity and altered the ubiquitination pathway in our ex vivo model}

Then, we investigated the curcumin effect on the SUMOylation and ubiquitination molecular pathways in our ex vivo model finding that protein SUMO-1ylation was increased in the retina lysates at $24 \mathrm{~h}(2.44 \pm 0.20, P<0.001$ vs $t 0$; Fig. $7 \mathrm{~A})$, but the pretreatment of both doses of curcumin was not able to significantly modify the SUMO-1ylation level.

A similar trend was also observed for UBC9 that was significantly increased at time $24(2.77 \pm 0.19, P<0.0001$; Fig. $7 A)$, but curcumin was inefficient to counteract this modification, and actually $10 \mu \mathrm{M}$ of curcumin increased the level of UBC9 even more than $24 \mathrm{~h}$ $(10 \mu \mathrm{M}$ : $5.61 \pm 0.25, P<0.0001$; Fig. $7 \mathrm{~A})$. The expression level of 
A

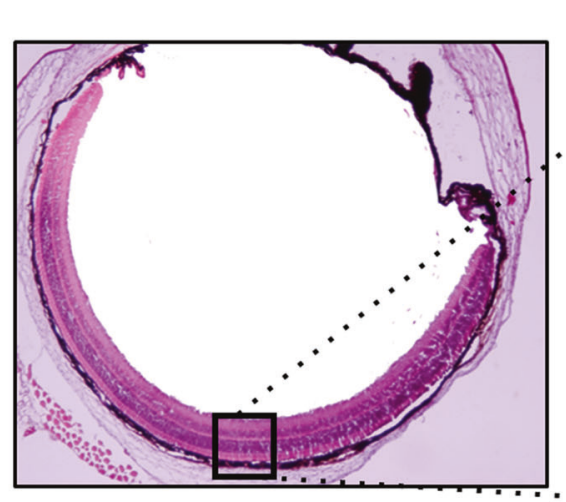

B

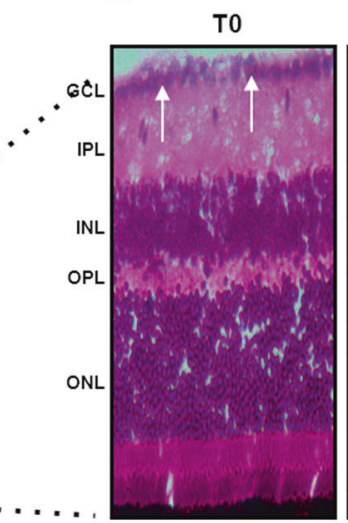

c

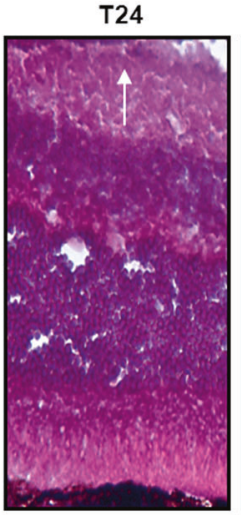

D

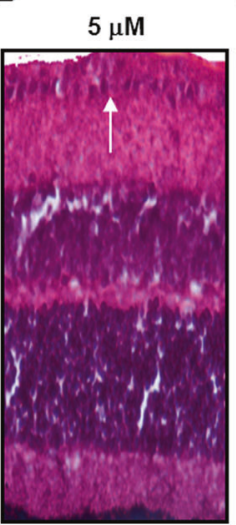

E

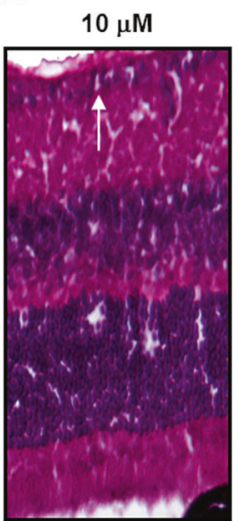

F

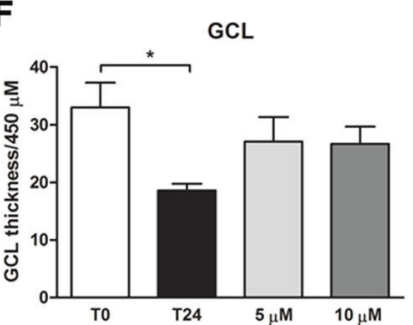

I

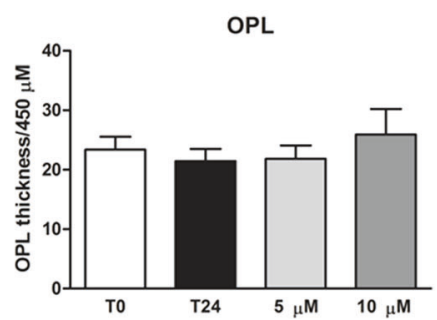

G

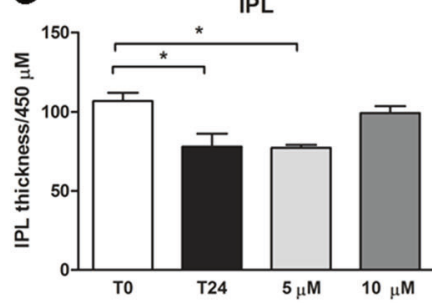

J

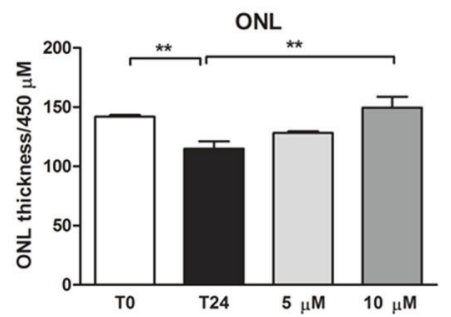

H

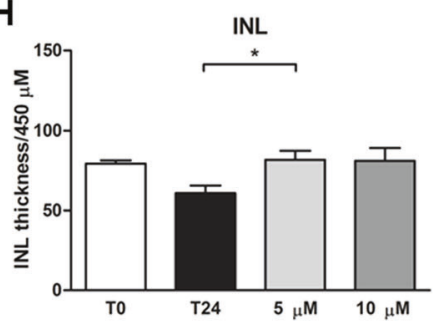

K

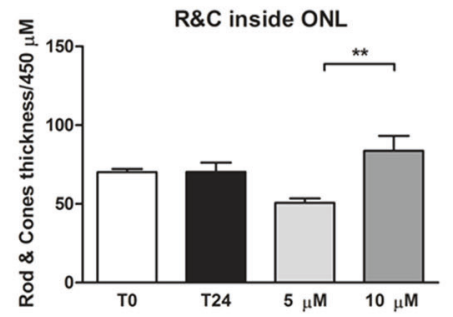

Fig. 5 Alterations of retinal layers thickness and influence of curcumin administration in induced retinal degeneration ex vivo model. A-E Representative image of hematoxylin \& eosin (H\&E) eye's sections collected at time $0(\mathbf{A})$ and magnification of retinal sections examined at time $0(B)$, time $24(C), 24 \mathrm{~h}$ pre-treated with $5 \mu \mathrm{M}(\mathbf{D})$, and $10 \mu \mathrm{M}(\mathbf{E})$ of curcumin depicting retinal morphological changes in $\mathrm{H} \& \mathrm{E}$ staining. $\mathrm{GCL}$ ganglion cell layer, IPL inner plexiform layer, INL inner nuclear layer, OPL outer plexiform layer, ONL outer nuclear layer, OS outer segment. Scale bar: $20 \mu \mathrm{m}$. F-K Measurement of retinal layer thickness in H\&E eye sections collected at time 0 , after $24 \mathrm{~h}$ from eye removal, $24 \mathrm{~h}$ pretreated with 5 and $10 \mu \mathrm{M}$ of curcumin. Graphs indicated at time 24 a reduction of ganglion cell layer (GCL, F), inner plexiform layer (IPL, G), inner nuclear layer (INL, H), outer nuclear layer (ONL, J), if compared to time 0 . A tendency of increasing thickness was observed in samples pre-treated with curcumin in each layer analyzed, but resulted significant only for INL and ONL analysis (H, J); graph related to rod and cones inside ONL (R\&C, K) indicated a significant increase between 5 and $10 \mu \mathrm{M}$ curcumin-treated samples. The measurement of outer plexiform layer (OPL, I) did not show any significant differences among groups examined. ${ }^{*} P<0.05$ and ${ }^{* *} P<0.01[n=5]$. Data were expressed as mean \pm SEM. One-way ANOVA, Tukey's post hoc test.

ubiquitin was significantly decreased at time $24(0.62 \pm 0.08, P<$ 0.01 vs t0; Fig. 7B) while it was significantly increased by $5 \mu \mathrm{M}$ $(1.60 \pm 0.08, P<0.0001$; Fig. $7 \mathrm{~B})$ and $10 \mu \mathrm{M}$ of curcumin $(1.82 \pm$ $0.08, P<0.0001$; Fig. 7B). We tested the effect of curcumin on autophagy system and we found that LC3BII/I ratio was strongly augmented at time $24(5.98 \pm 0.34, P<0.0001$; Fig. 7B) and this effect was prevented by both 5 and $10 \mu \mathrm{M}$ of curcumin administration $(5 \mu \mathrm{M}: 2.42 \pm 0.34, P<0.0001 ; 10 \mu \mathrm{M}: 1.86 \pm 0.36$, $P<0.0001$ vs t24; Fig. 7B).

\section{DISCUSSION}

In this paper we described an interesting and fast method to study the RGC loss occurring when eyeballs are kept in PBS at $4{ }^{\circ} \mathrm{C}$ for $24 \mathrm{~h}$ after being enucleated from mice, procedure that includes the ONC. We demonstrated that in $24 \mathrm{~h}$ several biochemical parameters related to cells neurodegeneration were altered and curcumin, a turmeric bioactive compound, was efficient to partially contrast the retinal cell loss. Indeed, after $24 \mathrm{~h}$ from ONC the optic nerve lysate showed an increase of Caspase-3 and a drop of NeuN levels while retinal lysates showed an increase level of Caspase-9. This let us to suppose that ONC and eyeballs enucleation induced the onset of the apoptotic process which especially leads to RGC loss [39]. Within only $24 \mathrm{~h}$ from the ONC and eye isolation a huge increase of BCL2 level, a pro-apoptotic protein, was found in retinal lysate correlated to a significant reduction of $B C L-X L$, an anti-apoptotic protein, which confirms the activation of apoptotic pathway as already shown for RGCs [40]. Caspase- 3 has been indicated as mediator for RGC death following axotomy in vivo [41] while Caspase-7 is involved in an earlier step of the apoptotic signaling cascade in retinal degenerative model [27]. Caspase-7 was found activated in RGCs starting from $12 \mathrm{~h}$ post optic nerve crush reaching the plateau after 3 days [27], while Caspase-3 activation was delayed [30]. In our hands, both biochemical and immunofluorescence analysis suggest that our method developed predominantly an important RGC loss, as also 
A

T0 T24 $5 \mu \mathrm{M} \quad 10 \mu \mathrm{M}$

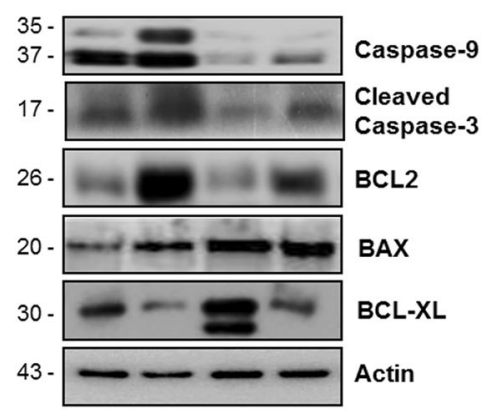

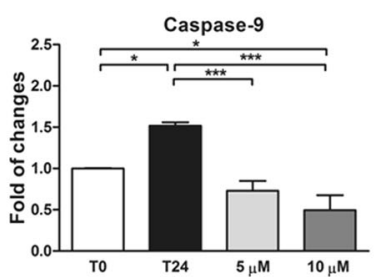

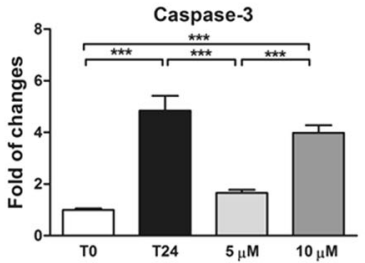

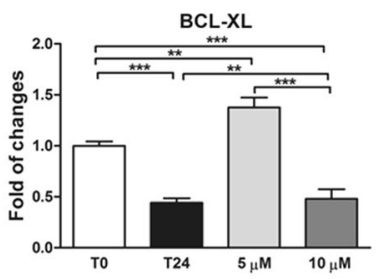

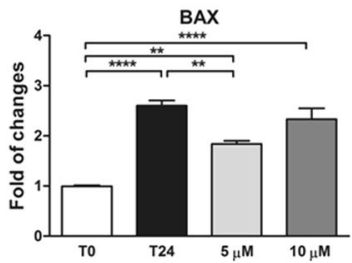

B

T0 T24 $5 \mu \mathrm{M} 10 \mu \mathrm{M}$

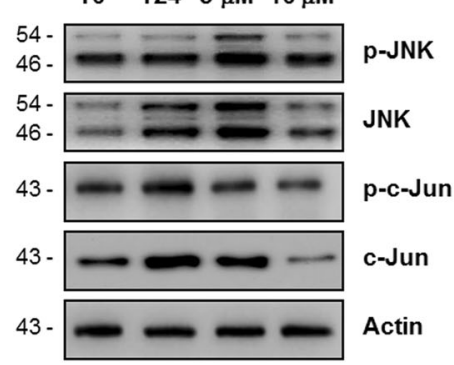

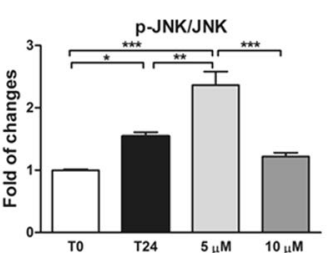

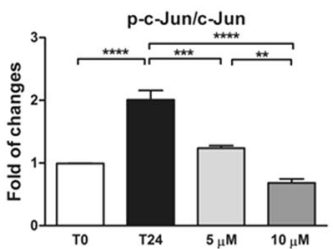

C T0 T24 $5 \mu \mathrm{M} 10 \mu \mathrm{M}$
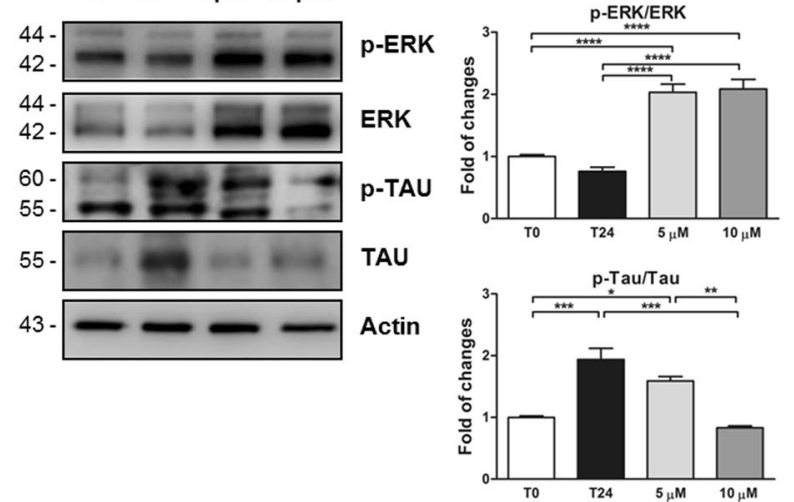

Fig. 6 Curcumin treatment prevents apoptotic and MAPK pathways activation in ex vivo model of retinal degeneration. A Representative western blots and relative quantifications showed the reduction of Caspase-9, cleaved-Caspases-3, BCL2, and BAX levels in retina homogenates caused by the $24 \mathrm{~h}$ pre-treatment of curcumin $(5$ and $10 \mu \mathrm{M})$ if compared to induced retinal degeneration at time 24 . The BCLXL level was significantly increased by $5 \mu \mathrm{M}$ of curcumin administration. ${ }^{*} P<0.05,{ }^{* *} P<0.01,{ }^{* * *} P<0.001$, and ${ }^{* * * *} P<0.0001$ [ $n=5$ ]. Data were expressed as mean \pm SEM. One-way ANOVA, Tukey's post hoc test. B, C Representative western blots and relative quantifications of JNK, c-Jun, ERK, and Tau. B Graphs indicated a significant augmented p-JNK/JNK and p-c-Jun/C-Jun ratios at time 24, reduced by curcumin administration; $5 \mu \mathrm{M}$ of curcumin significantly increase $\mathrm{p}$-JNK/JNK ratio if compared to other experimental conditions. C A significant decrease of $p$-ERK/ERK ratio at time 24 was caused by 5 and $10 \mu \mathrm{M}$ of curcumin administration. The augmented $\mathrm{p}$-Tau/Tau ratio observed at time 24 was reduced by curcumin treatment. ${ }^{*} P<0.05,{ }^{*} P<0.01,{ }^{* *} P<0.001$, and ${ }^{* * *} P<0.0001[n=5]$. Data were expressed as mean \pm SEM. One-way ANOVA, Tukey's post hoc test.

shown by the loss of BRN3A immunoreactions, a specific RGC marker.

Then, we found a powerful activation of JNK in retina lysates, which indicates its important role in cell apoptosis as previously described $[42,43]$.

JNK kinase regulates several transcription factors including CJun, but also many mitochondrial proteins like BCL2 and $B C L-X L$ $[18,44]$. As expected, being JNK phosphorylation induced by ONC at $24 \mathrm{~h}$ also a powerful c-Jun activation was observed, in line with the already known direct interaction cascade between p-JNK and p-c-Jun [45]. In fact, an alteration of BCL2 and BCL-XL levels was detected after $24 \mathrm{~h}$ from ONC, probably as a consequence of JNK and apoptosis cascade activation. Interestingly, the expression level of $B C L 2$ and $B C L-X L$ is opposite after $24 \mathrm{~h}$ from $\mathrm{ONC}$, in line with the already described interplay between the two proteins acting vicariously for each other in the anti-apoptotic system [46, 47]. The apoptotic induction at $24 \mathrm{~h}$ is able to induce the expression of a faint but visible band at around $26 \mathrm{kDa}$ which correspond to the pro-apoptotic isoform $\mathrm{BCL}-\mathrm{XS}[46,47]$. Moreover, a decreased activity of ERK, which counterbalances the JNK activation in apoptotic processes, was observed in the retinas collected after $24 \mathrm{~h}$ from $\mathrm{ONC}$, in line with published data $[48,49]$.

Interestingly, we observed an augmented Tau protein level as reported in animal models affected by Alzheimer's disease in which an aberrant accumulation of Tau protein in the retina is supposed to cause visual malfunction [18].

In our previous study we proposed a cooperative crosstalk among JNK, Tau, and SUMO-1 in oxidative stress condition [21] and accordingly these proteins have a role in retina function [50]; therefore, we examined the protein SUMO-1ylation in our ex vivo model. We found an increased SUMO-1ylated and UBC9 (SUMO-1 target enzyme) protein level with an opposite trend compared to ubiquitination level after $24 \mathrm{~h}$ from ONC which support the retinal suffering. In line with the observation that autophagy is activated in a model of optic nerve crush produced in rats [51] we found an augmented LC3B level in our model. The SUMOylation/ubiquitination process unbalance and the increase of the autophagic system activity suggest a potential engulfment of the degradation pathway which might be the cause for the pathological accumulation of Tau that leading consequently to the Tau hyper-phosphorylation and retinal cell death. 
A

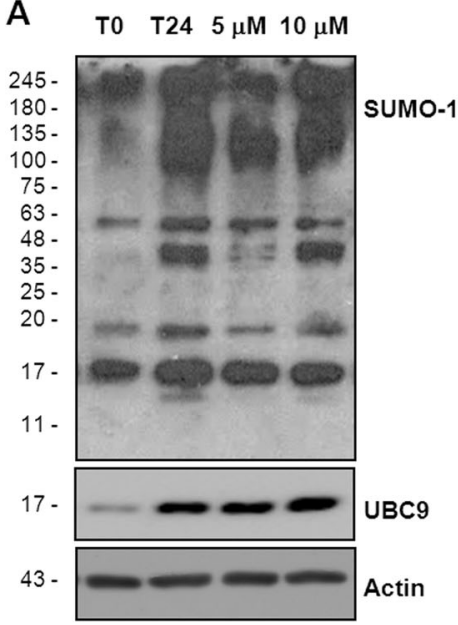

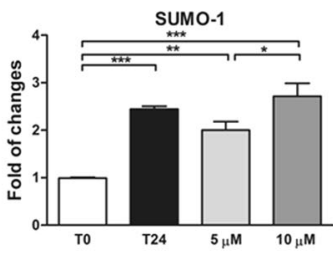

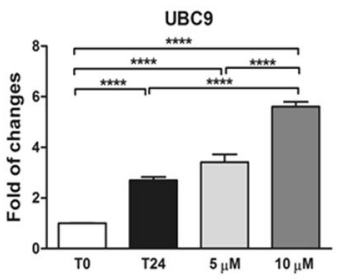

B

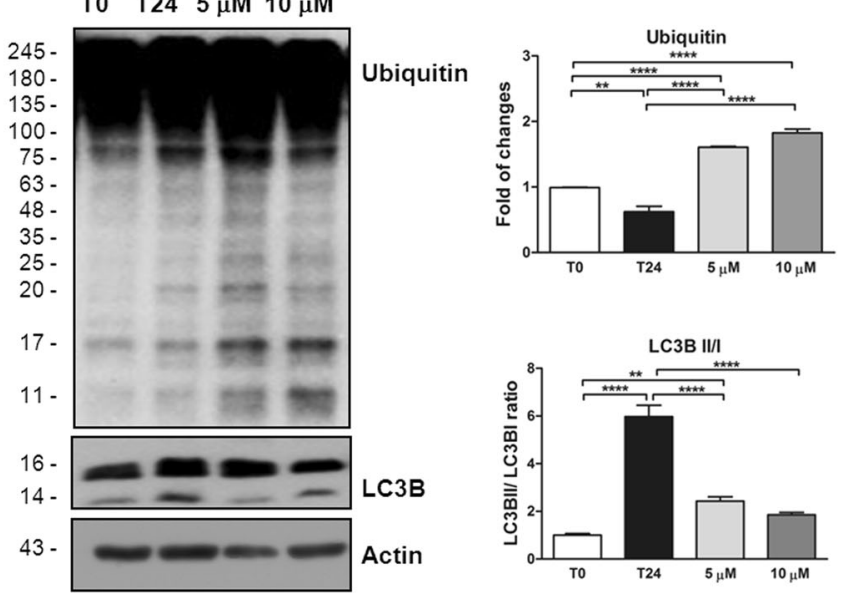

Fig. 7 Curcumin influences SUMO-1 and degradation pathway activity in ex vivo model of retinal degeneration. A-D Representative western blots and relative quantifications of SUMO-1, UBC9, Ubiquitin, and LC3B. A Graphs showed an increase of SUMO-1ylated proteins and UBC9 level at time 24 in retinas homogenates if compared to time 0 . The treatment with $10 \mu \mathrm{M}$ of curcumin induced a significant augmentation of SUMO-1 as well as UBC9 levels, while $5 \mu \mathrm{M}$ caused a slight reduction of SUMO-1 activity. B Histograms showed at time 24 a significant increase of Ubiquitin level and a reduction of LC3BII/I ratio induced by 5 and $10 \mu \mathrm{M}$ curcumin administration. ${ }^{*} P<0.05$, ${ }^{* *} P<0.01$, ${ }^{* * *} P<0.001$, and ${ }^{* * *} P<0.0001[n=5]$. Data were expressed as mean \pm SEM. One-way ANOVA, Tukey's post hoc test.

Therefore, we tested curcumin as potential neuroprotective molecule against degenerative processes in this ex vivo model. The therapeutic role of curcumin in retinal diseases has been already hypothesized $[25,26]$ and we also demonstrated, in a neuroblastoma cell line, that it is able to prevent the SUMO1-JNK-Tau axis hyperactivation in oxidative stress condition [21]. Indeed, a pre-treatment with 5 and $10 \mu \mathrm{M}$ of curcumin was able to counteract the activation of the apoptotic pathway, reducing Caspase- 9 and Caspase- 3 as well as apoptotic (BCL2 and BAX) markers and increased the anti-apoptotic (BCL-XL) level [21]. Specifically, although BCL-XS is activated by pro-apoptotic signals, the treatment with $5 \mathrm{uM}$ of curcumin was able to protect the retinal cells inducing a strong $B C L-X L$ expression. On the other hand, $10 \mu \mathrm{M}$ of curcumin exerted its retinal neuroprotective effect inducing an augmented expression level of BCL2 protein which vicariate the anti-apoptotic activity of BCL-XL. Probably, curcumin is able to activate two different anti-apoptotic cascades or acts on the same one at different levels depending on the administered concentration.

The protective effects of curcumin against apoptosis and oxidative damage have been already described in different in vitro and in vivo models of glaucoma [52, 53]. In our experiments, curcumin was able to reduce RGC loss when RGCs were counted in NeuN staining and the RGC layer was measured in hematoxylin-eosin staining. As expected, the other retinal layers thickness was comparable with control in both $24 \mathrm{~h}$ and curcumin treated indicating that our ex vivo model based on ONC is mainly effecting RGC survival.

In line with our results, pre-clinical studies showed that curcumin administration ( 2.5 and $10 \mu \mathrm{M})$ both in in vitro and in vivo models attenuated RGC and amacrine cell loss [25]. Interestingly, we observed that curcumin seems to re-equilibrate SUMOylation/Ubiquitination balance. Moreover, the strong activation of the autophagic system detected was well prevented by curcumin treatment, suggesting its inhibitory potential on this pathway as already observed [54].

JNK/c-Jun activation and Tau protein level were reduced by curcumin treatment, while ERK activity was significantly increased as already reported in different models of retinopathies $[52,55,56]$. Ten micromolar of curcumin was able to prevent the JNK activation probably for its direct antagonist effect on this kinase [57] especially reducing the JNK3 pro-apoptotic isoform, which can also explain the prevention of the autophagy pathway activation [58]. Unexpectedly, an increased JNK activity was observed after $5 \mu \mathrm{M}$ of curcumin treatment leading us to hypothesize that curcumin lower concentration is not able to act on JNK pathway, but it may induce the upstream ERK cascade activation. Further studies are to need to better clarify the curcumin effect on the different players of the apoptotic pathway (BCL2, BCL-XL, BAX, ERK, JNK).

Moreover, the activity of curcumin on JNK, reduction of autophagy pathway, and Tau hyperactivation may leads to think could contrast Tau hyper-phosphorylation and eventually Tau accumulation on the retina as described in different pathologies including Alzheimer's disease [59] or glaucoma [33].

Our findings showed a simple and non-invasive ex vivo model in which the ONC could mimic the main apoptotic events and RGC loss in a short temporal window and that the protective activity of curcumin could be further investigated for eye's pathologies.

\section{MATERIALS AND METHODS}

\section{Animals}

In this study we used 2-month-old C57BL/6J male mice purchased from Jackson Laboratories, USA. Animals were housed (4 per group) in standard mouse cages, all with (hard wood shavings) as bedding material, ad libitum food and water with a regular 12:12 h light/dark cycle (lights on 07:00 a.m.), at a constant room temperature of $22 \pm 2{ }^{\circ} \mathrm{C}$ and relative humidity approximately $55 \pm 10 \%$.

\section{Ex vivo treatment}

Animals were sacrificed by cervical dislocation and eyes and optic nerves were harvested to investigate the time-dependent retina neurodegeneration and the effect of ex vivo treatments. The enucleated eyeballs were kept at $4{ }^{\circ} \mathrm{C}$ in PBS and retinal dissection has been performed immediately after the sacrifice (time 0) or after 6 (time 6), 12 (time 12), and 24 (time 24) hours from the sacrifice. In each experimental condition, retinas were collected $[60,61]$ and processed for biochemical analysis. At the same time points, total eyes were collected for immunohistochemistry and immunofluorescence analysis $(n=5)$. A different group of animals was dedicated to investigate the curcumin influence on retina neurodegeneration. After the sacrifice, eyeballs with optic nerves were harvested and treated with the vehicle dimethyl sulfoxide (DMSO) $(1 \mu \mathrm{l})$, curcumin dissolved in DMSO at the concentration of $5 \mu \mathrm{M}(1 \mu \mathrm{l})$ or curcumin dissolved in DMSO at the concentration of $10 \mu \mathrm{M}(1 \mu \mathrm{l})$. After $24 \mathrm{~h}$ of the treatment, retinas and optic nerves were isolated from the eyecup and processed for biochemical 
Table 1. Table of antibodies used in the experiments.

\begin{tabular}{|c|c|c|c|c|}
\hline Primary antibody & Host animal & $\begin{array}{l}\text { Diluition for } \\
\text { western blot }\end{array}$ & $\begin{array}{l}\text { Dilution for } \\
\text { immunofluorescence }\end{array}$ & Distributor, Cat, num. \\
\hline Anti-BAX & Rabbit & $1: 500$ & & Cell Signaling, Danvers, MA, USA. cat.\#5023 \\
\hline Anti-BCL-XL & Rabbit & $1: 1000$ & & Cell Signaling, Danvers, MA, USA. cat.\#2764 \\
\hline Anti-BRN3A & Mouse & $1: 1000$ & $1: 50$ & $\begin{array}{l}\text { Santa Cruz Biotechnology, Milan, Italy (C- } \\
20 \text { sc31984) }\end{array}$ \\
\hline $\begin{array}{l}\text { Anti-Cleaved- } \\
\text { Caspase-9 }\end{array}$ & Rabbit & $1: 1000$ & & Cell Signaling, Danvers, MA, USA. cat. \#20750 \\
\hline Anti-p-c-Jun & Rabbit & $1: 1000$ & & Cell Signaling, Danvers, MA, USA. cat.\#9164 \\
\hline Anti-c-Jun & Rabbit & $1: 1000$ & & Cell Signaling, Danvers, MA, USA. cat. \#9165 \\
\hline Anti-JNK & Rabbit & $1: 1000$ & & Cell Signaling, Danvers, MA, USA.cat. \#9252 \\
\hline Anti-NeuN & Rabbit & $1: 1000$ & $1: 200$ & Cell Signaling, Danvers, MA, USA. cat. \#24307 \\
\hline Anti-SUMO-1 & Mouse & $1: 500$ & & $\begin{array}{l}\text { Santa Cruz Biotechnology, Milan, Italy(D-11sc- } \\
\text { 5308) }\end{array}$ \\
\hline Anti-p-Tau & Mouse & $1: 1000$ & & Thermo Scientific cat.\#MN1020 \\
\hline Anti-Tau & Mouse & $1: 1000$ & & Thermo Scientific cat. \#MA5-12808 \\
\hline Anti-UBC9 & Rabbit & $1: 1000$ & & Cell Signaling, Danvers, MA, USA. cat. \#4786 \\
\hline Anti-Ubiquitin & Mouse & 1:1000 & & Cell Signaling, Danvers, MA, USA. cat. \#3933 \\
\hline
\end{tabular}

analysis ( $n=5$ for each experimental group). Total eyeballs were collected for immunohistochemistry and immunofluorescence analysis $(n=5)$. The definition of curcumin doses was reported by Buccarello et al. [21].

\section{Preparation of retinal and optic nerve lysates}

Retinas and optic nerves were collected and separately lysed in $93 \mu \mathrm{l}$ of Lysis Buffer solution (LB) composed by 1\% Triton X-100 (Sigma-Aldrich, Milan, Italy, nr 9002-93-1), a complete set of protease inhibitors (Complete, Roche Diagnostics, Basel, Switzerland) and phosphatase inhibitors (Sigma, St. Louis, MO), $N$-ethylmaleimide (Sigma-Aldrich, Milan, Italy, nr 128-53-0), a buffer containing the following components (mM): TRIS acetate, 20; sucrose, 0.27; EDTA, 1; EGTA, 1 ; Na Orthovanadate, 1; NaF, 50; Na Pyrophosphate, 5; Na $\beta$ - glycerophosphate, 10; and DTT, 1 (Sigma-Aldrich, Milan, Italy). Then samples were kept for $30 \mathrm{~min}$ on ice to allow protein extraction. Later a centrifugation step of $10 \mathrm{~min}$ at 10,000 r.p.m. was applied to the samples and the supernatant was collected and stored at $-20^{\circ} \mathrm{C}$ until needed.

\section{Western blot}

Retinal and optic nerve protein concentrations were quantified using the Bradford Assay (Bio-Rad Protein Assay 500-0006, Munchen, Germany); $40 \mu \mathrm{g}$ of extracted proteins were separated by $10 \%$ SDS polyacrylamide gel electrophoresis. PVDF membranes were blocked in Tris-buffered saline (5\% non-fat milk powder, $0.1 \%$ Tween $20,1 \mathrm{~h}$, room temperature). Primary antibodies (see Table 1) were diluted in the same buffer (incubation overnight, $4^{\circ} \mathrm{C}$ ) and described in Table 1 . Blots were developed using horseradish peroxidase-conjugated secondary antibodies (anti-Mouse or anti-Rabbit, 1:5000; Santa Cruz Biotechnology, Milan, Italy) and the immunoreactive bands were visualized by exposure to the ECL chemiluminescence system (Cyanagen, westar antares nr XLS142, Bologna, Italy). Actin was used as a loading control for SUMO-1, UBC9, Caspase-3, and Ubiquitin levels. To quantify JNK, c-Jun, ERK activation, and Tau levels we examined the ratio between the phosphorylated and total kinase itself $(p-$ JNK/JNK, p-c-Jun/c-Jun, and p-ERK/ERK ratio) and the phosphorylated/total protein level ( $p$-Tau/Tau ratio). LC3B was quantified analyzing the ratio between LC3BII and LC3BI isoforms. Western blots were quantified by densitometry using ImageJ software and was based on at least three independent experiments.

\section{Immunohistochemistry and immunofluorescence analysis}

After the sacrifice, the eyes of the animals were fixed in cold methanol: acetic acid:PBS (3:1:4) overnight $[62,63]$ and were incubated overnight in $30 \%$ sucrose before being embedded within optimal cutting temperature compound (OCT, Sigma, St. Louis, MO, USA). Eyes were sectioned at a thickness of $12 \mu \mathrm{m}$ and eyeballs sections containing the optic disk (four slices per mouse) were used for subsequent analysis. For immunohistochemistry analysis, retinal crio-sections were mounted and stained with hematoxylin-eosin (H\&E) (Sigma- Aldrich, see ref. 64), cover-slipped with Eukitt, and observed under a light-transmission microscope (Nikon) [64].

Cell density in the GCL was determined for each eye by counting the number of cells in the middle part of retina over a distance of $300 \mu \mathrm{m}$ (200-500 $\mu \mathrm{m}$ from the edge of the optic disc). The thickness of each layers was also evaluated using ImageJ software and compared between eyes harvested at time 0 , time 24, and for each curcumin treatment. For immunofluorescence analysis, retinal crio-sections were permeabilized with PBS-Triton X-100 (Fluka) $0.5 \%$ for $15 \mathrm{~min}$, washed three times in PBS $1 \times$, and exposed in a blocking solution for $1 \mathrm{~h}$ in a humid chamber.

The primary antibody anti-BRN3a and anti-NeuN (see Table 1) was added and incubated overnight at $4{ }^{\circ} \mathrm{C}$. The following day, retinal sections were incubated with Alexa-488 (Green) (1:500; Invitrogen-Thermo Fisher, Milan, Italy) in PBS for 45 min in a humid chamber. Nuclear staining was performed with DAPI (1:500; Invitrogen-Thermo Fisher, Milan, Italy). Coverslips were mounted in Fluorsave mounting medium (Calbiochem, Millipore, Billerica, MA, USA, nr 345789). Positive cells were counted over a distance of $450 \mu \mathrm{m}$ and four consecutive sections were analyzed [65]. Images were acquired with a Olympus microscope equipped with a Olympus Confocal scan unit (microscope BX61 and Confocal system FV500) managed by AnalySIS Fluoview software with three lasers line, UV diode laser $(405 \mathrm{~nm}), \mathrm{Ar}-\mathrm{Kr}(488 \mathrm{~nm}), \mathrm{He}-\mathrm{Ne}$ green $(546 \mathrm{~nm})$, respectively, used to detect DAPI staining and secondary antibodies. Identical exposure 
time was used for all the samples. Images measures and analysis were performed by ImageJ software.

\section{Data analysis}

The experiments were repeated at least three times. Statistical analysis was performed using Graph Pad Prism 9 program. WB data and different neuronal counts were analyzed using one-way ANOVA, followed by Tukey's post hoc test. All data were expressed as mean \pm SEM with statistical significance given at $P<0.05$.

\section{DATA AVAILABILITY}

The datasets presented in this study can eventually required to the authors. The article has also Supplementary Material.

\section{REFERENCES}

1. Smith CA, Vianna JR, Chauhan BC. Assessing retinal ganglion cell damage. Eye Lond Engl. 2017;31:209-17.

2. Moore DL, Goldberg JL. Four steps to optic nerve regeneration. J. Neuroophthalmol. 2010;30:347-60.

3. Laha B, Stafford BK, Huberman AD. Regenerating optic pathways from the eye to the brain. Science . 2017;356:1031-4.

4. Cajal SR y, May RM. Cajal's degeneration and regeneration of the nervous system. In: DeFelipe J, Jones EG, editors. Oxford University Press; 1991 [cited 2020 Nov 16]. Available from: https://oxford.universitypressscholarship.com/view/10.1093/ acprof:oso/9780195065169.001.0001/acprof-9780195065169.

5. Quigley HA. The number of people with glaucoma worldwide in 2010 and 2020 . Br. J. Ophthalmol. 2006;90:262-7.

6. Mantravadi AV, Vadhar N. Glaucoma. Prim Care Clin Off Pract. 2015;42:437-49.

7. McKinnon SJ, Schlamp CL, Nickells RW. Mouse models of retinal ganglion cell death and glaucoma. Exp Eye Res. 2009;88:816-24.

8. Pang $\mathrm{I}-\mathrm{H}, \mathrm{Clark}$ AF. Rodent models for glaucoma retinopathy and optic neuropathy. J. Glaucoma. 2007;16:483-505.

9. Lindsey JD, Weinreb RN. Elevated intraocular pressure and transgenic applications in the mouse. J Glaucoma. 2005;14:318-20.

10. Morrison JC, Johnson E, Cepurna WO. Rat models for glaucoma research. Prog. Brain Res. 2008;173:285-301.

11. Morrison JC, Guo WOCY, Johnson EC. Pathophysiology of human glaucomatous optic nerve damage: insights from rodent models of glaucoma. Exp. Eye Res. 2011;93:156-64.

12. Barres BA, Silverstein BE, Corey DP, Chun LL. Immunological, morphological, and electrophysiological variation among retinal ganglion cells purified by panning. Neuron 1988;1:791-803.

13. Yin Y, Cui Q, Gilbert H-Y, Yang Y, Yang Z, Berlinicke C, et al. Oncomodulin links inflammation to optic nerve regeneration. Proc Natl Acad Sci USA. 2009;106:19587-92.

14. Yin Y, Henzl MT, Lorber B, Nakazawa T, Thomas $T$, Jiang F, et al. Oncomodulin is a macrophage-derived signal for axon regeneration in retinal ganglion cells. Nat Neurosci. 2006;9:843-52.

15. Johnson TV, Tomarev SI. Rodent models of glaucoma. Brain Res Bull. 2010;81:349-58.

16. Kikuchi M, Tenneti L, Lipton SA. Role of p38 mitogen-activated protein kinase in axotomy-induced apoptosis of rat retinal ganglion cells. J Neurosci. 2000;20:5037-44.

17. Sun $X-Y$, Tuo Q-Z, Liuyang Z-Y, Xie A-J, Feng X-L, Yan X. et al. Extrasynaptic NMDA receptor-induced tau overexpression mediates neuronal death through suppressing survival signaling ERK phosphorylation. Cell Death Dis. 2016;7:e2449

18. Buccarello L, Sclip A, Sacchi M, Castaldo AM, Bertani I, ReCecconi A, et al. The c-jun $\mathrm{N}$-terminal kinase plays a key role in ocular degenerative changes in a mouse model of Alzheimer disease suggesting a correlation between ocular and brain pathologies. Oncotarget. 2017;8:83038-51.

19. Luo J-M, Cen L-P, Zhang X-M, Chiang SW, Huang Y, Lin D, et al. PI3K/akt, JAK/STAT and MEK/ERK pathway inhibition protects retinal ganglion cells via different mechanisms after optic nerve injury: Mechanism of pathway inhibition-induced survival. Eur J Neurosci. 2007;26:828-42.

20. Marigo V. Programmed cell death in retinal degeneration: targeting apoptosis in photoreceptors as potential therapy for retinal degeneration. Cell Cycle. 2007;6:652-5.

21. Buccarello L, Dragotto J, lorio F, Hassanzadeh K, Corbo M, Feligioni M. The pivotal role of SUMO-1-JNK-Tau axis in an in vitro model of oxidative stress counteracted by the protective effect of curcumin. Biochem Pharmacol. 2020;178:114066 https://doi.org/10.1016/j.bcp.2020.114066.
22. Abad-Morales V, Domenech EB, Garanto A, Marfany G. mRNA expression analysis of the SUMO pathway genes in the adult mouse retina. Biol Open. 2015;4:224-32.

23. Wright LE, Frye JB, Gorti B, Timmermann BN, Funk JL. Bioactivity of turmericderived curcuminoids and related metabolites in breast cancer. Curr Pharm Des. 2013;19:6218-25.

24. Beatty $\mathrm{S}$, Koh $\mathrm{H}-\mathrm{H}$, Phil M, Henson D, Boulton $\mathrm{M}$. The role of oxidative stress in the pathogenesis of age-related macular degeneration. Surv Ophthalmol. 2000;45:115-34.

25. Burugula B, Ganesh BS, Chintala SK. Curcumin attenuates staurosporinemediated death of retinal ganglion cells. Invest Ophthalmol Vis Sci. 2011;52:4263-73.

26. López-Malo D, Villarón-Casares CA, Alarcón-Jiménez J, Miranda M, Díaz-Llopis $M$, Romero FJ. et al. Curcumin as a therapeutic option in retinal diseases. Antioxidants (Basel). 2020;9:48.

27. Choudhury S, Liu Y, Clark AF, Pang I-H. Caspase-7: a critical mediator of optic nerve injury-induced retinal ganglion cell death. Mol Neurodegener. 2015;10:40.

28. Kim R. Unknotting the roles of $\mathrm{Bcl}-2$ and $\mathrm{BCl}-\mathrm{xL}$ in cell death. Biochem Biophys Res Commun. 2005;333:336-43.

29. Yue J, LÇpez JM. Understanding MAPK signaling pathways in apoptosis. Int J Mol Sci. 2020;21:2346.

30. Brentnall M, Rodriguez-Menocal L, De Guevara R, Cepero E, Boise LH. Caspase-9, caspase-3 and caspase-7 have distinct roles during intrinsic apoptosis. BMC Cell Biol. 2013;14:32.

31. Levkovitch-Verbin H. Retinal ganglion cell apoptotic pathway in glaucoma: initiating and downstream mechanisms. Prog Brain Res. 2015;220:37-57.

32. Levkovitch-Verbin H, Dardik R, Vander S, Melamed S. Mechanism of retinal ganglion cells death in secondary degeneration of the optic nerve. Exp Eye Res. 2010;91:127-34.

33. Chiasseu M, Alarcon-Martinez L, Belforte N, Quintero H, Dotigny F, Destroismaisons $\mathrm{L}$, et al. Tau accumulation in the retina promotes early neuronal dysfunction and precedes brain pathology in a mouse model of Alzheimer's disease. Mol Neurodegener. 2017;12:58.

34. LaBonne C. SUMO weighs in on a photoreceptor finish. Dev Cell. 2009;16:165-6.

35. Feligioni M, Nisticò R. SUMO: a (oxidative) stressed protein. Neuromol Med. 2013;15:707-19.

36. Lobanova ES, Finkelstein S, Li J, Travis AM, Hao Y, Klingeborn M, et al. Increased proteasomal activity supports photoreceptor survival in inherited retinal degeneration. Nat Commun. 2018;9:1738.

37. Uğuz AC, Öz A, Nazıroğlu M. Curcumin inhibits apoptosis by regulating intracellular calcium release, reactive oxygen species and mitochondrial depolarization levels in SH-SY5Y neuronal cells. J Recept Signal Transduct. 2016 ;36:395-401.

38. Wu L, Jiang C, Kang Y, Dai Y, Fang W, Huang P. Curcumin exerts protective effects against hypoxia-reoxygenation injury via the enhancement of apurinic/apyrimidinic endonuclease 1 in SH-SY5Y cells: involvement of the PI3K/AKT pathway. Int J Mol Med. 2020;45:993-1004.

39. Sánchez-Migallón MC, Valiente-Soriano FJ, Nadal-Nicolás FM, Vidal-Sanz M, Agudo-Barriuso M. Apoptotic retinal ganglion cell death after optic nerve transection or crush in mice: delayed RGC loss with BDNF or a caspase 3 inhibitor. Invest Ophthalmol Vis Sci. 2016;57:81-93.

40. Maes ME, Schlamp CL, Nickells RW. BAX to basics: how the BCL2 gene family controls the death of retinal ganglion cells. Prog Retin Eye Res. 2017;57:1-25.

41. Kermer $P$, Klöcker N, Labes $M$, Thomsen S, Srinivasan A, Bähr M. Activation of caspase-3 in axotomized rat retinal ganglion cells in vivo. FEBS Lett. 1999;453:361-4.

42. Chen YR, Tan $\mathrm{TH}$. The c-Jun $\mathrm{N}$-terminal kinase pathway and apoptotic signaling (review). Int J Oncol. 2000;16:651-62.

43. Dhanasekaran DN, Reddy EP. JNK signaling in apoptosis. Oncogene 2008;27:6245-51.

44. Cui J, Zhang M, Zhang Y-Q, Xu Z-H. JNK pathway: diseases and therapeutic potential. Acta Pharmacol Sin. 2007;28:601-8.

45. May GH, Allen KE, Clark W, Funk M, Gillespie DA. Analysis of the interaction between c-Jun and c-Jun N-terminal kinase in vivo. J Biol Chem. 1998;273:33429-35.

46. Schott AF, Apel IJ, Nuñez G, Clarke MF. Bcl-XL protects cancer cells from p53mediated apoptosis. Oncogene 1995;11:1389-94.

47. Masui T, Hosotani R, Ito D, Kami K, Koizumi M, Mori T, et al. Bcl-XL antisense oligonucleotides coupled with antennapedia enhances radiation-induced apoptosis in pancreatic cancer. Surgery. 2006;140:149-60.

48. Xia Z, Dickens M, Raingeaud JL, Davis RJ, Greenberg ME. Opposing effects of ERK and JNK-p38 MAP kinases on apoptosis. Science. 1995;270:1326-31.

49. Valter K, Bisti S, Gargini C, Di Loreto S, Maccarone R, Cervetto L, et al. Time course of neurotrophic factor upregulation and retinal protection against light-induced damage after optic nerve section. Invest Ophthalmol Vis Sci. 2005;46:1748-54. 
50. Zhang L, Li DW. SUMOylation regulation of retina development and functions. Curr Mol Med. 2016;16:803-8.

51. Kang L-H, Zhang S, Jiang S, Hu N. Activation of autophagy in the retina after optic nerve crush injury in rats. Int J Ophthalmol. 2019;12:1395-401.

52. Farajipour H, Rahimian S, Taghizadeh M. Curcumin: a new candidate for retinal disease therapy? J Cell Biochem. 2019;120:6886-93.

53. Yue Y-K, Mo B, Zhao J, Yu Y-J, Liu L, Yue C-L, et al. Neuroprotective effect of curcumin against oxidative damage in BV-2 microglia and high intraocular pressure animal model. J Ocul Pharmacol Ther. 2014;30:657-64.

54. Shakeri A, Cicero AFG, Panahi $Y$, Mohajeri M, Sahebkar A. Curcumin: a naturally occurring autophagy modulator. J Cell Physiol. 2019;234:5643-54.

55. Bian ZM, Elner VM, Yoshida A, Kunkel SL, Elner SG. Signaling pathways for glycated human serum albumin-induced IL- 8 and MCP-1 secretion in human RPE cells. Invest Ophthalmol Vis Sci. 2001;42:1660-8.

56. Wang S, Ye Q, Tu J, Zhang M, Ji B. Curcumin protects against hypertension aggravated retinal ischemia/reperfusion in a rat stroke model. Clin Exp Hypertens. 2017;39:711-7.

57. Chen YR, Tan TH. Inhibition of the c-Jun N-terminal kinase (JNK) signaling pathway by curcumin. Oncogene. 1998;17:173-8.

58. Zhou Y-Y, Li Y, Jiang W-Q, Zhou L-F. MAPK/JNK signalling: a potential autophagy regulation pathway. Biosci Rep. 2015;35.

59. den Haan J, Morrema THJ, Verbraak FD, de Boer JF, Scheltens P, Rozemuller AJ, et al. Amyloid-beta and phosphorylated tau in post-mortem Alzheimer's disease retinas. Acta Neuropathol. Commun. 2018;6:147.

60. Escher P, Schorderet DF. Exploration of the visual system: Part 1: Dissection of the mouse eye for RNA, protein, and histological analyses. Curr Protoc Mouse Biol. 2011;1:445-62.

61. Sondereker KB, Stabio ME, Jamil JR, Tarchick MJ, Renna JM. Where you cut matters: a dissection and analysis guide for the spatial orientation of the mouse retina from ocular landmarks. J Vis Exp. 2018;57861..

62. Krebs MP, Collin GB, Hicks WL, Yu M, Charette JR, Shi LY, et al. Mouse models of human ocular disease for translational research. PLoS ONE. 2017;12:e0183837.

63. Tisi A, Flati V, Delle Monache S, Lozzi L, Passacantando M, Maccarone R. Nanoceria particles are an eligible candidate to prevent age-related macular degeneration by inhibiting retinal pigment epithelium cell death and autophagy alterations. Cells. 2020;9:1617.

64. Fischer $\mathrm{AH}$, Jacobson KA, Rose J, Zeller R. Hematoxylin and Eosin staining of tissue and cell sections. Cold Spring Harb Protoc. 2008;2008:pdb.prot4986-pdb. prot4986.

65. Mead B, Thompson A, Scheven BA, Logan A, Berry M, Leadbeater W. Comparative evaluation of methods for estimating retinal ganglion cell loss in retinal sections and wholemounts. PLoS ONE. 2014;9:e110612.

\section{ACKNOWLEDGEMENTS}

This work was funded by "Fondo Ordinario Enti" (FOE, D.M 865/2019) funds in the framework of a collaboration agreement between the Italian National Research Council and EBRI (2019-2021).

\section{AUTHOR CONTRIBUTIONS}

LB designed, performed and analyzed the experiments and drafted the manuscript. JD helped to perform and analyze the experiments. KH, MC, RM and MF reviewed the manuscript. MF conceived and supervised the project and found the financial support. All authors have read and approved the manuscript.

\section{COMPETING INTERESTS}

The authors declare no competing interests.

\section{ETHICS STATEMENT}

The study was conducted according to the guidelines of the national and international laws and policies (EU Directive 2010/63/EU for animal experiments) and approved by the Ethical Committee on animal experiments of EBRI "Rita LeviMontalcini" Foundation (Rome, Italy). The scientific project was approved by the Italian Ministry of Health (Permit Number F8BBD.N.7OK).

\section{ADDITIONAL INFORMATION}

Supplementary information The online version contains supplementary material available at https://doi.org/10.1038/s41420-021-00760-1.

Correspondence and requests for materials should be addressed to Lucia Buccarello or Marco Feligioni.

Reprints and permission information is available at http://www.nature.com/ reprints

Publisher's note Springer Nature remains neutral with regard to jurisdictional claims in published maps and institutional affiliations.

\begin{abstract}
cc) Open Access This article is licensed under a Creative Commons adaptation, distribution and reproduction in any medium or format, as long as you give appropriate credit to the original author(s) and the source, provide a link to the Creative Commons license, and indicate if changes were made. The images or other third party material in this article are included in the article's Creative Commons license, unless indicated otherwise in a credit line to the material. If material is not included in the article's Creative Commons license and your intended use is not permitted by statutory regulation or exceeds the permitted use, you will need to obtain permission directly from the copyright holder. To view a copy of this license, visit http://creativecommons. org/licenses/by/4.0/.
\end{abstract}

(c) The Author(s) 2021 